\section{Behavioral Assessment of the Dysexecutive Syndrome}

Helga Peter ${ }^{1}$ und Thomas Penzel ${ }^{2}$

${ }^{1}$ Marburg, Deutschland

${ }^{2}$ Interdisziplinäres Schlafmedizinisches Zentrum, Charité -

Universitätsmedizin Berlin, Berlin, Deutschland

\section{Definition}

Verhaltensdiagnostik der Handlungsausführungsstörung. Siehe $\triangleright$ „Leistungs-, Schläfrigkeits- und Vigilanzmessung".

\title{
Synonyme
}

BADS 